\title{
Editorial: Multimedia and Social Data Processing in Vehicular Networks
}

\author{
Qing Yang ${ }^{1} \cdot$ Tigang Jiang ${ }^{2} \cdot$ Wenjia $\mathrm{Li}^{3} \cdot$ Guangchi Liu $^{4} \cdot$ Danda B. Rawat ${ }^{5} \cdot \mathrm{Jun} \mathrm{Wu}^{6}$
}

Published online: 14 December 2019

(C) Springer Science+Business Media, LLC, part of Springer Nature 2019

\section{Editorial:}

As the growth of auto industry and wireless communication technologies, vehicular computing and communication devices have been widely installed on automobiles in recent years. Multimedia and social data processing in vehicular network, however, is facing several technical challenges. First, despite of the growing computing capability, vehicular multimedia and social applications still need to be carefully designed such that the resources being used and the associated network traffic can be minimized. Second, current vehicular network bandwidth is far less than the data generation rate of most vehicular multimedia applications. How to enable efficient communication among multimedia applications of different cars becomes acritical technical issue. In the end, the multimedia and social data generated by vehicles contain tons of hidden information.

Qing Yang

Qing.Yang@unt.edu

1 Department of Computer Science and Engineering, University of North Texas, 155 Union Cir, Denton, TX 76203, USA

2 Department of Information and Communication Engineering, University of Electronic Science and Technology of China (UESTC), 4 E 2nd Section, 1st Ring Rd, Jianshe Road, Chengdu, China

3 New York Institute of Technology, New York, NY, USA

4 Research and Development Department, Stratifyd, Inc., Charlotte, NC, USA

5 Cyber-security and Wireless Networking Innovations (CWiNs) Research Lab, Howard University, 2400 6th St NW, Washington, DC 20059, USA

6 College of Electronics and Information Engineering, Tongji University, 1238 Gonghexin Rd, Zhongshan North Road, Shanghai, China
This special issue features five selected papers with high quality. The first article, "The Signal Control Optimization of Road Intersections with Slow Traffic Based on Improved PSO", authored by Hongluan Zhao, Guoyong Han, Xiaofei Niu, established a multi-objective signal timing optimization model about the vehicle average delay, slow traffic delay, vehicle stopping times and traffic capacity; furthermore, used an improved particle swarm optimization algorithm (PSO) to solve it.

The second article titled "An Anomaly Recognition and Autonomic Optimization Method to User's sequence behaviors for D2D communications in MCC", presented a recognition method to user abnormal behaviors for D2D communications in mobile cloud environment from the perspective of user trustability, covering hierarchical matching, blacklist mechanism, maximum and right-most path extension.

In the next article with the title "Physical Layer Security Performance of Mobile Vehicular Networks", authors investigated the physical layer security performance of the mobile vehicular networks over N-Nakagami fading channels in order to overcome the challenges of the mobile vehicular networks, such as broadcast nature of wireless channels, vehicular user mobility, and the diversity of vehicular network structures.

As an important component for vehicular network, road infrastructure network plays an important role in transportation. Reasonable road network density and traffic demand work as a good foundation for easing the traffic pressure. The fourth article titled "Estimating the Floor Area Ratio of the Vehicular Infrastructure Network Based on Road Grid Cell" proposed a model to estimate the floor area ratio upper limit from the perspective of traffic demand-supply equilibrium, designed an automatic tool based on a typical scenario, set up some parameters to do numerical simulation.

The last article titled "Socially Aware Security Message Forwarding Mechanism in VANETs" proposed a security 
message forwarding mechanism for vehicular ad hoc networks (VANETs) to deal with the security challenges of VANETs, such as data privacy protection, data security transmission, vehicular relationship aware, vehicular trust assessment and trust forwarding decision.

Acknowledgements The guest editors are thankful to our reviewers for their effort in reviewing the manuscripts. We also thank the Edit-in-Chief, Dr. Imrich Chlamtac for his supportive guidance during the entire process.

Publisher's note Springer Nature remains neutral with regard to jurisdictional claims in published maps and institutional affiliations.

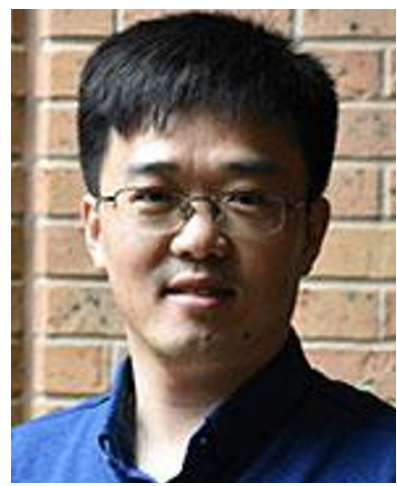

Qing Yang, Ph.D, is an assistant professor in the Department of Computer Science

and Engineering at University of North Texas. He received B.S. and M.S. degrees in Computer Science from Nankai University and Harbin Institute of Technology, China, in 2003 and 2005, respectively. He received his Ph.D degree in Computer Science from Auburn University in 2011. His research interests include Internet of Things, autonomous vehicular system, network

security and privacy.

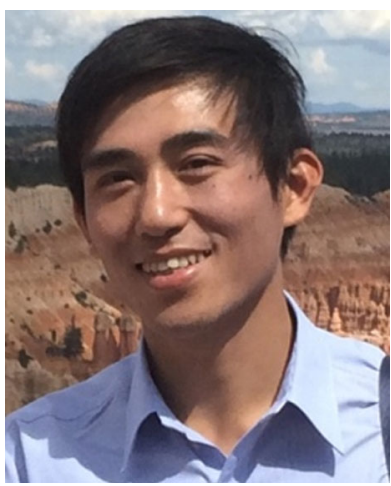

Tigang Jiang is currently an associate professor in the Department of Information and Communication Engineering at University of Electronic Science a n d T e h n olog of China(UESTC), also he is the vice director of the lab of Internet of Things and Multimedia (IOTMM), UESTC. $\mathrm{He}$ received B.S. degree in Material Science from Xi'an Jiaotong University, China, in 1998. He received M.S. and $\mathrm{Ph} . \mathrm{D}$ degrees in Computer Science from Shouthwest Jiaotong University, China, in 2001 and 2005, respectively. From 2014 to 2015, He joined Computer Communication Research Group (CCRG) as a visiting scholar of Computer Engineering Department, University of California, Santa Cruz (UCSC). His current research interests include computer networks, Internet of Things, cognitive radio networks, deep learning, artificial intelligence and $3 \mathrm{D}$ reconstruction.

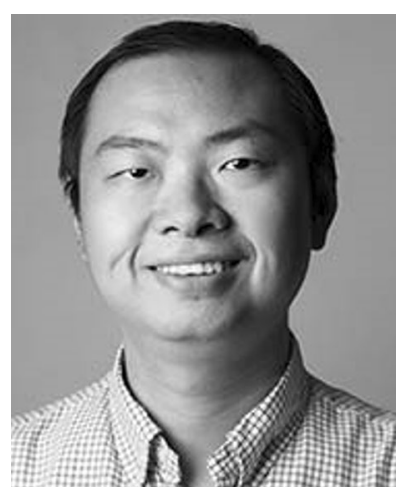

Wenjia Li specializes in cyber security, computer networks, and mobile computing, especially security, trust, and privacy issues in wireless networks, cyber-physical systems (CPS) such as intelligent transportation systems, internet of things (IoT), and mobile social networks. Prior to joining NYIT in 2014, he was a tenure-track assistant professor at Georgia Southern University, a public comprehensive university within the University System of Georgia (USG). He obtained his Ph.D. in computer science from University of Maryland Baltimore County (UMBC) in August 2011, and a master's degree in computer science and a bachelor's degree in telecommunication engineering from Hunan University, China, in 2005 and 2002, respectively.

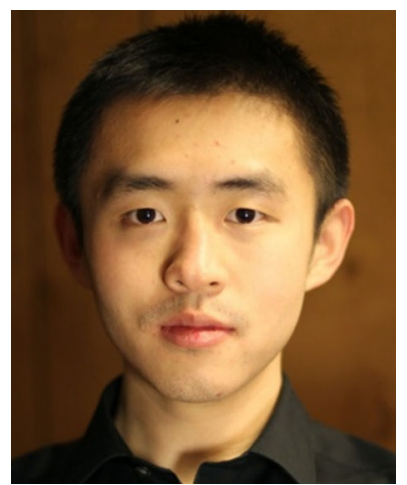

Guangchi Liu is currently a research scientist in the research \& development department of Stratifyd, Inc., Charlotte, NC, USA. He received his Ph.D. in Computer Science from Montana State University, USA. His research interests include Internet of things, trust assessment, social network, and wireless sensor network. 


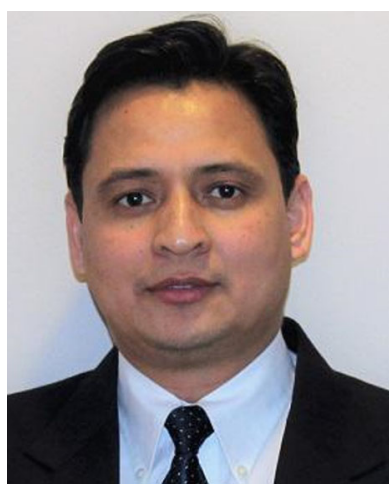

Danda B. Rawat is an Associate Professor in the Department of Electrical Engineering \& Computer Science, Founding Director of the Data Science and Cybersecurity Center (DSC2), Graduate Program Director of Howard-CS Graduate Programs, Director of Graduate Cybersecurity Certificate Program and Founding Director of Cyber-security and Wireless Networking Innovations (CWiNs) Research Lab at Howard University, Washington, DC, USA. Dr. Rawat is engaged in research and teaching in the areas of cybersecurity, machine learning and wireless networking for emerging networked systems including cyber-physical systems, Internet-of-Things, smart cities, software defined systems and vehicular networks. His professional career comprises more than 15 years in academia, government, and industry.

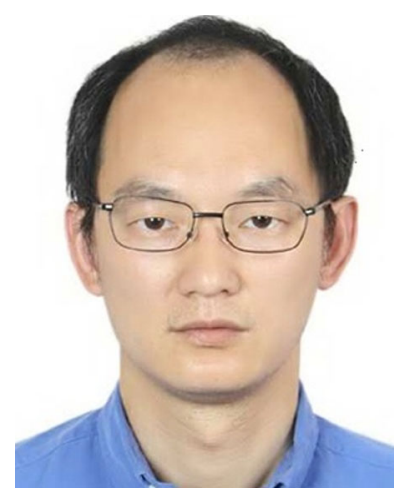

Jun $\mathrm{Wu}$ received the B.S. degree in information engineering and the M.S. degree in communication and electronic systems from Xidian University, Xian, China, in 1993 and 1996, respectively, and the Ph.D. degree in signal and information processing from the Beijing University of Posts and Telecommunications, Beijing, China, in 1999.,He was a Principal Scientist with Huawei, Shenzhen, China, and Broadcom, Irvine, CA, USA. He joined the College of Electronics and Information Engineering, Tongji University, Shanghai, China, as a Professor, in 2010. 